\title{
Analyzing the influence of the disk motion on longitudinal oscillations of a beam with rheological properties
}

\author{
Maria Kalmova ${ }^{1, *}$, and Georgy Pavlov ${ }^{1}$ \\ ${ }^{1}$ Samara State Technical University, Academy of Architecture and Civil Engineering, \\ Molodogvardeyskaya St., 194, Samara, 443001, Russia
}

\begin{abstract}
The paper gives the analysis of the influence of longitudinal oscillations of a beam, which is caused by a circular hard disk motion along the beam, on the mode of disk motion. The problem of rolling the disk on the plane in its various formulations has been investigated by different researchers as well as by the authors of this paper. The paper considers two variants of properties of the beam material: an elastic beam and a viscoelastic beam, which fits the Kelvin rheological model and has relaxation and creep properties. When solving the problem, the Fourier method is used as a method of separation of variables. When examining the beam, and assuming it as hereditary deformable, a rheological force of the reaction is introduced, this depends on both on longitudinal strains and on the rate of these strains The result is presented as functions of time which are adaptable for numerical integration. It is shown that oscillations of a rheological beam are produced by a moving disk and can be considered as self-oscillations.
\end{abstract}

\section{Introduction}

Today various authors [1-6] regularly consider the problems of the motion of rotation bodies with rheological properties. The analysis of rotation bodies motion, where the stability of bodies motion is also investigated [7-12], is given. Although the rheological models have a long history, there are still many unsolved issues. That seems interesting to estimate the effect of longitudinal strains of rigidly fixed rod, which material is modelled by the Kelvin relaxation body, on the disc dynamics.

\section{Materials and methods}

The disk motion along the beam is determined by two coordinates - $\xi(t)$ and $\varphi(t)$ Figure 1.

\footnotetext{
*Corresponding author: kalmova@inbox.ru
} 


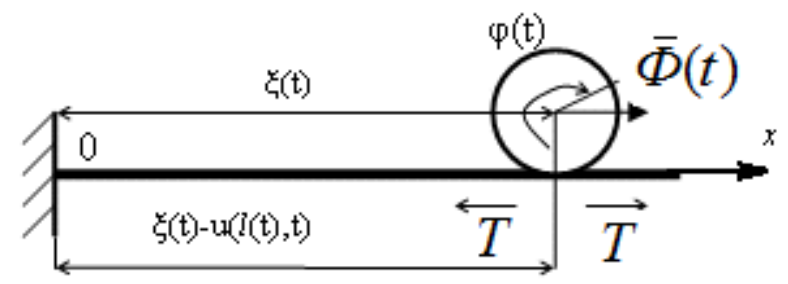

Fig. 1. Rigidly fixed rod.

Let us denote the Langrangian coordinate of contact point of the disk with the beam by $l(t)$, i.e. $x_{c}=l(t)$. Then we have the following expression

$$
\xi(t)=l(t)+u(l(t), t)
$$

which represents the geometric constraint equation. The equation seems correct

$$
R \dot{\varphi}=\dot{l}(t)\left(1+\frac{\partial u(l, t)}{\partial x}\right),
$$

which can be considered, as a non-linear non-holonomic constraint at the contact point of the disk with the beam [1-5]. Active force $\bar{\Phi}(t)$ effects on the disk, driving it into motion and being applied to the centre of mass, as well as at the point of contact the reaction force $\mathrm{T}$, directed along the beam and caused by longitudinal deformation of the beam. Let us write the initial system of equations

$$
m \ddot{\xi}(t)=\Phi(t)-T, \Im \ddot{\varphi}(t)=T \cdot R, \rho \frac{\partial^{2} u}{\partial t^{2}}-E \frac{\partial^{2} u}{\partial x^{2}}=\delta(x-l(t) T
$$

Here $\delta(x-l)-$ Dirac delta function.

Simplifying the problem, let us consider the regime of uniform motion of a disk, linearize the non-holonomic constraint equation, and we obtain

$$
R \Omega t=l(l), \Phi=T, \Omega \text { - angular velocity of the disk. }
$$

The solution of the equation of longitudinal oscillations will be tried as the sum of two functions $u(x, t)=v(x, t)+w(x, t)$. The function $v(x, t)$ must satisfy the homogeneous equation $\frac{\partial^{2} v}{\partial t^{2}}=a^{2} \frac{\partial^{2} v}{\partial x^{2}}$ and meet the conditions

$$
\left.v\right|_{t=0}=f(x),\left.\frac{\partial v}{\partial t}\right|_{t=0}=F(x),\left.v\right|_{x=0}=\left.\dot{v}\right|_{x=l}=0,
$$

where $a=\sqrt{\frac{E}{\rho}}$ 
The function $w(x, t)$ must satisfy the non-homogeneous equation $\frac{\partial^{2} w}{\partial t^{2}}=a^{2} \frac{\partial^{2} w}{\partial x^{2}}+\delta(x-l(t)) T$ and meet zero initial and boundary conditions

$$
\left.w\right|_{t=0}=\left.\frac{\partial w}{\partial t}\right|_{t=0}=0,\left.w\right|_{x=0}=\left.\dot{w}\right|_{x=l}=0
$$

The solution of the free oscillation equation (4) is the function

$$
u(x, t)=\sum_{k=0}^{\infty}\left(a_{k} \cos \frac{k \pi a t}{l}+b_{k} \sin \frac{k \pi a t}{l}\right) \sin \frac{k \pi x}{l},
$$

where $\quad a_{k}=\frac{2}{l} \int_{0}^{l} f(x) \sin \frac{k \pi x}{l} d x, b_{k}=\frac{2}{k \pi a} \int_{0}^{l} F(x) \sin \frac{k \pi x}{l} d x$.

The function $w(x, t)$ will be sought in the form of a series by own functions $\sin \frac{k \pi x}{l}$ in the homogeneous problem

$$
w(x, t)=\sum_{k=1}^{\infty} \gamma_{k}(t) \sin \frac{k \pi x}{l}
$$

Let us consider the case when the differential equations for describing unknown functions $\gamma_{k}(t)$ are as follows

$$
\ddot{\gamma}_{k}(t)+\omega^{2} \gamma_{k}(t)=q_{k}(t), \text { where } q_{k}(t)=\Phi \sum_{k=1}^{\infty} \delta\left(x-R \Omega \tau_{k}\right), \omega=\frac{\pi a}{2 l}
$$

This is the fundamental oscillation of the beam. We apply to this equation the integral Laplace transform $[5,6]$. We obtain the equation relative to the image $\Gamma(s) \doteqdot \gamma(t)$

$$
\left(s^{2}+\omega^{2}\right) \Gamma(s)=\frac{4}{k \pi} \Phi \sum_{k=0}^{\infty} \int_{0}^{\infty} e^{-s t} \delta(x-R \Omega \tau k) d t
$$

Since $\int_{-\infty}^{t} \delta(s-R \Omega \tau k) d s=\eta(x-R \omega \tau k)$, i.e. $\dot{\eta}(x-R \Omega \tau k)=\delta(s-R \Omega \tau k)$, where $\eta$ Heaviside step function, but by the property of the original $\eta(x-R \Omega \tau k) \doteqdot e^{\frac{-s R \Omega \tau k}{s}}$, then $\delta(x-R \Omega \tau k)=\dot{\eta}(x-R \Omega \tau k) \doteqdot s \frac{e^{-s R \Omega \tau}}{s}=e^{-s R \Omega \tau k}$.

Therefore $\int_{-\infty}^{t} e^{-s t} \delta(s-R \Omega \tau k) d t=e^{-s R \Omega \tau k}, \sum_{k=1}^{\infty} e^{-s R \omega \tau k}=\left(1-e^{-s R \Omega \tau}\right)^{-1}$ 
by virtue of which Laplace transform is of the form $\Gamma(s)=\frac{\Phi}{\pi\left(s^{2}+\omega^{2}\right)\left(1-e^{-s R \Omega \tau}\right)}$.

Let us find the original $\gamma(t) \doteqdot \Gamma(s)$. The function $\gamma$ is meromorphic, and it has poles at the points $s_{1,2}= \pm i \omega$ and $s_{k}=2 R \Omega \pi i$. If $\tau$ is not integral multiple $\tau_{1}=2 \pi / \omega$, then all poles are simple and we get

$$
\gamma(t)=\frac{\Phi}{\omega^{2}}\left(\frac{1}{\tau}-\frac{\omega}{2 \sin (\omega \tau / 2)} \cos \omega\left(t+\frac{\tau}{2}\right)-\sum_{k=1}^{\infty} \frac{2 \tau}{\tau^{2}-k^{2} \tau_{1}^{2}} \cos k \frac{\tau_{1}}{\tau} \omega t\right) .
$$

Substituting the function $\gamma(t)$ in (7) we find the law of forced longitudinal oscillations of a beam [7-9].

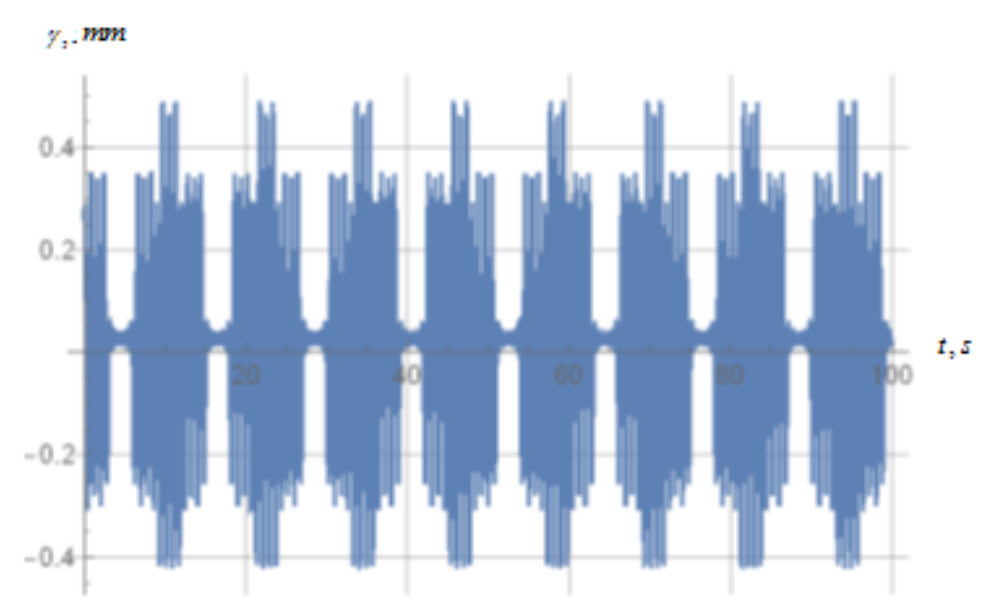

Fig. 2. The graph of forced oscillations in case of an elastic beam.

Now consider the effect of small strains of a beam on the character of the motion of a disk along the beam, provided with hereditary properties on the motion mode of the disk along the beam.

There is rheological reaction force at the point of contact of the disk with the beam

$$
P(t)=E\left(x(t)-\int_{0}^{t} \mathfrak{R}(t-\tau) x(\tau) d \tau\right)
$$

where $\mathfrak{R}(t-\tau)=\frac{E-\bar{E}}{n E} e^{-\frac{t-\tau}{n}}$ - relaxation kernel of material, $E, \bar{E}$ - long-term and instantaneous modulus of elasticity, $n$ - relaxation time.

This force is the source of longitudinal strains of a beam. Then the equation (8) is as follows

$$
\ddot{\gamma}_{k}(t)+\omega^{2} \gamma_{k}(t)=E \sum_{k=0}^{\infty} \delta(\gamma-R \Omega t)\left[\gamma_{k}(t)-\Omega^{2} \int_{0}^{t} \mathfrak{R}(t-\tau) \gamma_{k}(\tau) d \tau\right]+\Phi
$$


Here $\Omega$ - angular velocity of the disk. The solution of the equation (14) is tried applying Laplace transforms for zero and boundary conditions

$$
\begin{gathered}
L\left\{\gamma_{k}(t)\right\}=\Gamma(s), \\
L\left\{\ddot{\gamma}_{k}(t)\right\}=s^{2} \Gamma(s), \\
L\{\delta(\gamma-R \Omega k \tau)\}=e^{-s R \Omega k \tau}, \\
L\left\{\Omega^{2} \int_{0}^{t} \Re(t-\tau) \gamma_{k}(\tau) d \tau\right\}=\Omega^{2} \Gamma(s) \bar{\Gamma}(s), \text { where } \bar{\Gamma}(s)=\frac{E-\bar{E}}{n E} \frac{1}{\frac{1}{n}+s}
\end{gathered}
$$

After having applied Laplace transforms, taking into account (15), we find that $\Gamma(s)=\frac{\Phi}{s^{2}+\omega^{2}+E\left(1-\Omega^{2} F(s)\right)(s R \Omega \tau-1)}$. Applying the method of undetermined coefficients, we find the residues of the poles. Then the original $\gamma(t)$ is as follows $\gamma(t)=e^{-0.48 t}(6 \cos 22,9 t+4 \sin 22,9 t)+e^{-0,1 t}$.

The pattern of the rheological process is illustrated in figure 3.

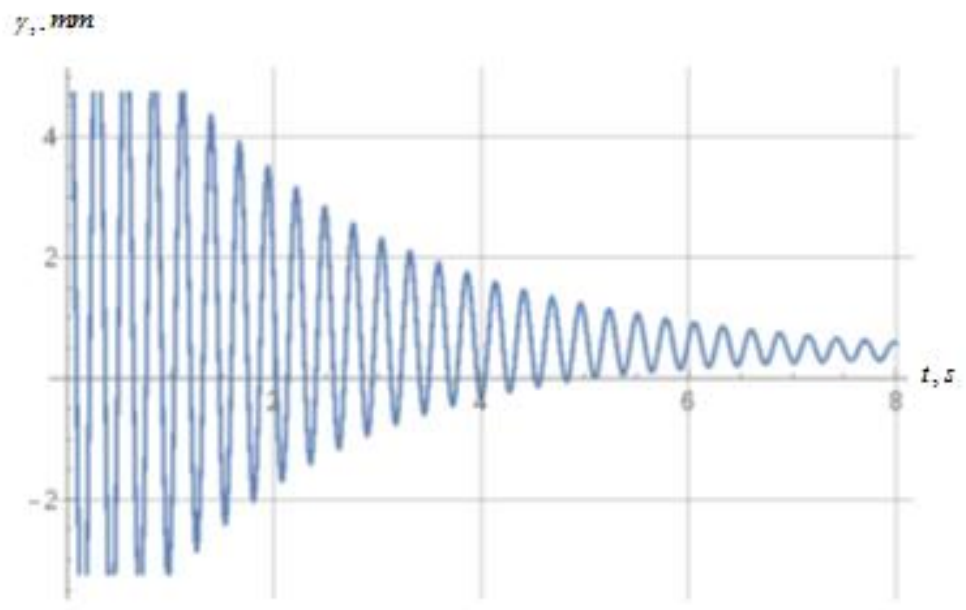

Fig. 3. The graph of forced oscillations of a beam with rheological properties.

\section{Results}

The result of the work can be the obtained graphs of forced oscillations of a beam, in case of an elastic beam and a beam with rheological properties. The graphs indicate that taking into account the longitudinal oscillations of a beam considerably increases the period of oscillations. The obtained results can be used in solution of direct and inverse problems in structural mechanics and in solution of parametric optimization problems [13-18]. 


\section{Conclusions}

In conclusion, our vision of the mechanism of the force interaction of a disk with a beam can be outlined. The moment of viscous friction, imposing restrictions on the rotational motion of the disk creates conditions for a series of instantaneous-consecutive elementary motions. This leads to instantaneous elementary tension-compression strains of a beam. Therefore, instantaneous elastic force appears in a beam preventing the strains. As soon as instantaneous elastic force exceeds the level of viscous friction forces, instantaneous motion of beam fibre goes in the reverse direction, eliminating strains of the beam. This process repeats over as the disk, affected by the stated force, moves. Thus, the longitudinal oscillations of a beam in this case can be considered as self-oscillations.

\section{References}

1. Y. Rabotnov, Elements of hereditary mechanics of solid bodies (1977)

2. A. Rzhanitsyn, Creep theory (1968)

3. O. Goroshko, Vestnik of the Kiev University 25, 51 (1983)

4. F. Badalov, A. Abdukarimov, B. Hudoyarov, Computational technologies, 12(4), 17 (2007)

5. A. Ishlinsky, Izvestiya USSR Academy of Sciences. TSD 3, 24 (1945)

6. A. Rzhanitsyn, Issues of system mechanics deformed in time (1949)

7. A. Ishlinsky, V. Antufyev, et al., Casebook on the strength of materials, theory and illustrative examples (2001)

8. F. Badalov, Dynamic vibration dampers of heredity-strained systems (2003)

9. G. Pavlov, Problems of nonholonomic mechanics (2014)

10. G. Pavlov, M. Kalmova, Vestnik of Tomsk State University 3(19), 68 (2012)

11. G. Pavlov, M. Kalmova, Vestnik of Samara State Technical University. Physical and mathematical sciences Series 2(19), 186 (2009)

12. G. Pavlov, M. Kalmova, A. Pischulev, D. Panfilov, IOP Conf. Series: Earth and Environmental Science 87, 082037 (2017)

13. V. Alpatov, MATEC Web of Conferences 117, 00007 (2017)

14. V. Alpatov, A. Sakharov, and etc., MATEC Web Conf. 86, 02021 (2016)

15. V. Alpatov, A. Belyakova, Traditions and innovations in construction and architecture. Construction, 35 (2016)

16. V. Alpatov, A. Lukin, Procedia Engineering 111, 20 (2015)

17. V. Alpatov, A. Mushkat, Industrial and civil construction 8, 31 (2010)

18. I. Kholopov, V. Alpatov, Issues of optimal structural design. Collection of papers of IV all-Russia seminar, 24 (2002) 